\title{
An Object Oriented Multi-dimensional Model for Intelligent Substation Information Integration
}

\author{
Tongwei $\mathrm{Yu}$ \\ State Grid Electric Power Research Institute of \\ Liaoning Provincial Power Company \\ Shenyang, China \\ e-mail: yu_twei@hotmail.com \\ Yundong Cao \\ Shenyang University of Technology \\ Shenyang, China \\ e-mail: ydcaosyut@163.com
}

\begin{abstract}
Object oriented algorithm is used for intelligent substation information process, based on XML Schema. One of the most important sessions for the intelligent substation construction is to integrate the mass historical data of devices and to establish the multi-dimensional model for OLAP or data mining. Considering the different requirements on substation devices' data from variety departments, this article introduces an object oriented multidimensional model, which could provide a better interaction with SCL. Intelligent substation is an object-oriented data model, in the process of integration platform construction, the establishment of multidimensional data model is the follow-up on-line analytical processing (OLAP) and data mining of the prerequisites. This paper puts forward an object-oriented multidimensional data model can be solved by sending the data type of the differences caused by different devices "impedance mismatch" problem, and in system upgrades, extensions will also has a certain advantage.
\end{abstract}

Keywords-intelligent substation; object oriented; multidimensional model; XML Schema; SCL

\section{INTRODUCTION}

Intelligent substation is an important node for the construction of the smart grid [1]. In the structure of future power system, Substation data will become the key technology of intelligent information processing. The data model of intelligent substation (SCL) will use the XML Schema to definite the file syntax structure [2]. Substation configuration description language is a kind of describe the structure of intelligent electronic devices, parameters, the communication topology, the interval structure of switch and the file format of the relationship between them [3]. The International Electro technical Commission (IEC TC57) technical committee officially released international standard about substation IED equipment interconnection IEC61850 in 2004,which involves the information model and service specifications in substation, the substation communication mapping and system integration, to protect and control the design and produce of the automation products and the substation automation system, and this

\author{
Juyong Cui \\ Shenyang University of Technology \\ Shenyang, China \\ e-mail: cuijuyong_sy@126.com
}

implementation will have guiding significance, become the international standard of interconnection of equipments in the substation IED.

Intelligent substation mainly includes two parts of intelligent high voltage equipment and substation unified information platform. Intelligent high voltage equipment mainly includes intelligent transformer, intelligent high voltage switch equipment, and electronic transformer. Intelligent transformer and control systems rely on communication optical fiber is connected, can grasp the state of transformer parameters and operating data. When the change of operation mode, equipment according to the system voltage, power, decide whether to adjust the tap; when the equipment problems, will issue a warning and provide conditions, reduce operational costs, to a certain extent, reduce the risk, improve the operation reliability of transformer. Intelligent high voltage switch equipment is switch equipment and control equipment with high performance, equipped with electronic devices, sensors and actuators, with monitoring and diagnosis function. The electronic transformer is refers to the pure optical transformer, magneto optic glass transformer, which can effectively overcome the disadvantages of the traditional electromagnetic transformer. Substation unified information platform has two functions, one is horizontal information sharing system, main performance is that the unified information obtained various upper applications management system; two is the system of longitudinal information standard, mainly for the transparency of each layer to the upper layer application support.

IEC 61850 specification for SCL language content, Language involves logical node, data collection, reporting, control block, connection, IP address and other communications within the station configuration. XML has become the standard of data description and exchange on the Internet, as a result, the XML format of SCL has made it simple for the substation system integration [5].

This article puts forward the object-oriented multidimensional data model has the following two advantages: it has good property in compatibility on the 
object-oriented data model of intelligent substation. The data structure of different equipment in intelligent substation is different, and the polymorphism of objectoriented feature can solve the problem of "impedance mismatch". Object-oriented technology has good interface for future system maintenance, upgrading and extension provides a convenient conditions.

Intelligent power grid construction is based on China's energy consumption and load distribution of regional distribution characteristics, to adapt to the power grid development taken to our current and future social development, on all kinds of energy, especially the largescale wind power and solar power are included in and out of strong adaptability, large scope, and can realize the efficient allocation of energy resources. The construction of smart grid in China has risen to the height of the national strategic level. Intelligent substation is one of the core platforms of energy conversion and control for strong smart grid construction, prospects remain bright.

Photovoltaic power generation is random, intermittent and volatility, which makes the grid capacity, is limited in a certain range, which to some extent limited the largescale photovoltaic applications. The initiative of smart grid is development to promote the social parties to use photovoltaic power generation, photovoltaic generation and development efforts to improve and use efficiency.

Our research on smart grid has been in progress. At present, the domestic power grid cannot satisfy the need of development of photovoltaic industry, so our country will be the construction of the "special high-voltage as the core" and "strong smart grid", in order to solve the problem of grid connected photovoltaic power, promote the use of new energy sources.

The smart grid is in UHV power network as the backbone grid, strong grid coordinated development of power grids at all levels as the foundation, use of communication, information and advanced control technology. To build a unified strong smart grid to information, automation, digital, interactive characterized.

The world of smart grid and new energy sources such as solar power have invested a lot of money in research, this is because the new energy power generation based on a smart grid development and development. Smart grid of the high-speed, reliable, economic and safe power transmission channel provides better guarantee delivery for photovoltaic power, its development and application is the complement and promotes each other.

\section{ADVANTAGES OF XML SCHEMA}

\section{A. Support for data types}

One of the XML Schema the most important ability is support for data types. Based on the data type support:

The content of the document can be more easily described allowed

Can be more easily verified the correctness of data; can be more easily worked together with the data from the database; can be more easily defined data constraint (data facets); can be more easily defined data model (or data); can be more easily converted the data in different data types.

\section{B. Use the XML grammar}

Another important feature of schema is about XML, which is written by XML. Prepared by the XMLXML Schema has a lot of advantages:

(a)Do not have to learn a new language.

(b)You can use the XML editor to edit the Schema file

(c)You can use the XML parser to parse the Schema file

(d)Through the XML DOM to process Schema

(e)Through the XSLT to Schema conversion

\section{Can protect data communication}

When the data from the sender to be sent to the receiving party, the key point is the both sides should have about the content the same "expected value". By XML Schema, the sender can use a recipient can understand the way to describe data.

\section{Scalability}

XML Schema is extensible, because they are written by XML. Through the Schema definitions can be extended, you can: reuse your Schema in other Schema.

Your own data types may be created by the derivative of the standard types, references to multiple Schemas in the same document.

\section{THE DATA WAREHOUSE AND MULTIDIMENSIONAL DATA MODEL}

\section{A. The Data Warehouse}

A data warehouse is a repository of information collected from multiple sources, stored in a consistent mode, usually resides in a site. Data warehouse through data cleaning, transformation, integration, and the process of loading and refreshed periodically to construct [6]. The framework of data warehouse is presented in Fig .1.

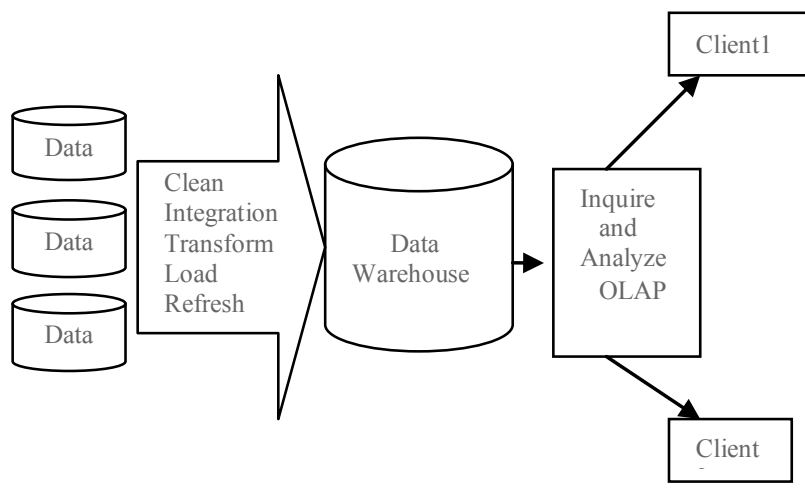

Figure 1. Classic framework data warehouse

\section{B. The Multidimensional Data Model}

Data warehouse need a concise, subject-oriented of the model, is advantageous for the OLAP processing analysis. At present, the most popular data warehouse data model is multidimensional model, the main form of model is a star schema, the snowflake schema and the fact constellation schema [6].

(a) Star schema

The star schema (also known as the star connection mode, data cube, or multidimensional model) is the most 
simple style data warehouse architecture. The star schema consists of one or more of the fact table is referenced in any number of dimension tables. The star schema is considered to be a special snowflake model important, and more efficient query processing is simple.

It contains a large amount of data in the fact table and a small data volume of dimension table. Each dimension in a table, according to each dimension table contains a set of attributes. The star schema in the application of data warehouse is the most commonly used data representation model, is also one of the highest operating cost in the OLAP queries [7].

(b) Snowflake schema

The snowflake model is a multidimensional database logical arrangement in the table, so that the entity relationship diagram is similar to the shape of the snow. Snowflake schema represents the fact table is connected to a plurality of layers. The snowflake model is similar to the star schema. However, in the snowflake schema, size normalized to multiple related tables and star pattern size of none standardized, each dimension table consists of a single. The complex shape of the snow appears, with dimension snowflake model, and has a hierarchy, and multiple sub table of the parent table effect will only affect the dimension table instead of the fact table.

If the star schema often merge together using the dimensions of regularization processing, the data further broken down into the attached table, get the snowflake model.

(c) Fact constellation schema

A conceptual model of a common data warehouse, this model is often applied to data relationships than the star type model and snowflake model more complicated situations. Fact constellation model requires multiple fact tables share dimension tables, and therefore can be regarded as a set of star model, so it is also called Galaxy model. This model is a combination of the both, breaking the limit of star schema has only one fact table, and the fact table sharing all or part of the dimension table.

\section{THE OBJECT-ORIENTED MULTIDIMENSIONAL DATA MODEL}

Object-oriented data structure can through these two fundamental elements, which are the object and class, with the aid of encapsulation, inheritance and combination mechanism to build a complex structure, data operation is done by method and message [8]. Object-oriented programming has become the dominant design method [9].

XML has gradually become the international standard of transmission and exchange of network data, whose advantage is that any system can be through the XML parser to read XML data and don't worry about the problem of system does not support.

\section{A. The ETL Process}

In the sending end (the source database), first will transmit data into XML data file, and then sent to the receiver through the network (data warehouse); after receiving side received the file, according to the corresponding XML Schema format data parsing into tree structure, which can be created the multidimensional data mode in the last. Literature [12] describes a structure of the
XML Schema import the framework of multidimensional data model.

Object-oriented of multidimensional data model refers to the fact table and dimension table of the model, using object-oriented inheritance features, to expand the certain data attributes in the table. Inherited part involves specific data attributes, which will not effect on the XML node, has no effect on the foreign key links of the multidimensional model. Source data import multidimensional model flow schematic diagram is presented in Fig .2.

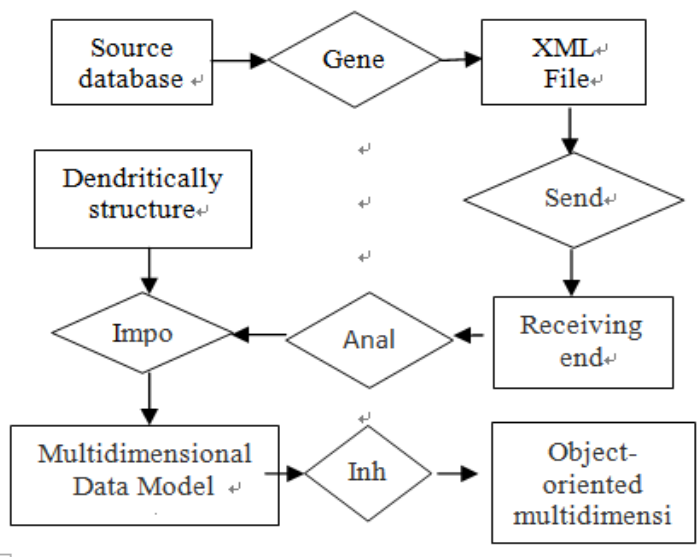

Figure 2. source data import multidimensional model flow schematic diagram

\section{B. Oriented Object Instances}

With a smart substation data as an example, multidimensional data model is used to support the objectoriented oracle $10 \mathrm{~g}$ environment to build.

Analog of XML Schema drive".

$<$ ? $\mathrm{xml}$ version $=$ " 1.0 " encoding $=$ " UTF -80 "? $>$

< xs: schema xmlns: xs = "http: / / www. w3. org/ 2001/ XML Schema">

$<$ xs: element name $=$ "HejiaData" >

$<$ xs: complexType $>$

$<$ xs: sequence $>$

$<$ xs: element name $=$ " Time" type $=$ "xs: date" $/>$

$<$ xs: element name $=$ "Location" type $=$ "xs: string" $/>$

$<$ xs: element name $=$ "Substation" type $=$ "xs: string" $/>$

$<$ xs: element name= "DeviceID" type = "xs: string"/ $>$

$</$ xs: sequence $>$

$<$ xs: attribute name $=$ "AnaValue" type= "xs: decimal"/ $>$

$<$ xs: attribute name $=$ "avgValue" type= "xs: decimal"/ $>$

$<$ xs: attribute name $=$ "MinValue" type $=$ "xs: decimal"/ >

$<$ xs: attribute name = "MaxValue" type= "xs: decimal"/ $>$

$</$ xs: complexType $>$

$</$ xs: element $>$

$</$ xs: schema $>$

Analog import to multidimensional data model, Schematic diagram of the multidimensional data model is presented in Fig .3. 


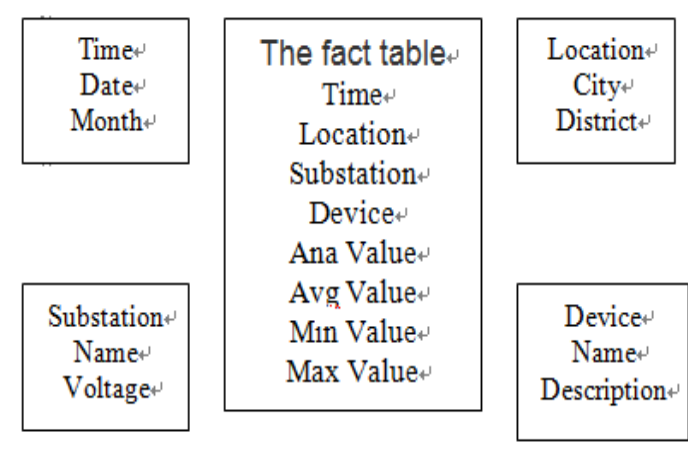

Figure 3. Schematic diagram of the multidimensional data model

And some of the logic variables only at state changes to send the data, in the XML Schema XS: attribute is often only one variable (value of 0 or 1 ). Then you need to use the object-oriented inheritance features to operate the multidimensional data model.

(a) Create an object-oriented classes; t_mdmodel;

CREATE TYPE $t$ mdmodel AS OBJECT(

Time TIMESTAMP,

Location VARCHAR2(20),

Substation VARCHAR(10),

Device VARCHAR2(40)

) NOT FINAL;

/

(b) Create two classes t_anadata and t_logicdata inheritance respectively;

CREATE TYPE t_anadata UNDER t_mdmodel(

AnaValue DECIMAL,

avgValue DECIMAL,

MinValue DECIMAL,

MaxValue DECIMAL );

CREATE TYPE $\mathrm{t}$ anadata UNDER $\mathrm{t}$-mdmodel( LogicValue SIGNTTYPE );

(c) Use the subclass object instead of the superclass object;

CREATE TABLE hejia_mdmodel OF t_mdmodel; INSERT INTO hejia mdmodel VALÜES(

$\mathrm{t}$ anadata("e 16-FEB-1210:00:00"e,

„Liaoning/Chaoyang,," Hejia ", „PROT/MMXU1

\$MX\$PhV\$phsC\$cVal\$mage, " "50.0"e, "50.0", "50.0

"e $\left.50.0^{\circ e}\right)$

);INSERT INTO hejia mdmodel VALUES(

t logicdata("e16-FEB-1210:05:04"e,

„Liaoning/Chaoyang,", Hejia" , „LD0

\section{/AlmGGIO1\$ST\$Alm1 ${ }^{\text {ee }}$ "e $1^{\text {ee }}$}

);

(d)When the query multidimensional data table, use VALUE keyword can see the entire set of properties all the elements in the table, can be based on it to slice and dice, roll-up and drill-down analysis and processing of data and the data mining operation.

\section{CONCLUSION}

Intelligent substation is an object-oriented data model, in the process of integration platform construction, the establishment of multidimensional data model is the follow-up on-line analytical processing (OLAP) and data mining of the prerequisites. This paper puts forward an object-oriented multidimensional data model can be solved by sending the data type of the differences caused by different devices "impedance mismatch" problem, and in system upgrades, extensions will also has a certain advantage. But object-oriented model for inheritance in the ETL phase, it could lead to extra time because of the inheritance operation and the consumption of resources, Such as real-time demand of system is higher, you will need to further optimize the process of ETL stage of data integration.

\section{REFERENCES}

[1] J.Feng,"Intelligent Substation Principle and Testing Technology.”Chi.Ele.Pow.Pre, vol.4 ,pp.11-14,May 2011.

[2] L.He,"IEC 61850 Introduction to the Application." Chi.Ele.Pow.Pre,vol.8 ,pp.24-28,. July 2012.

[3] J.Tan,"Intelligent Substation Technology and Practice." Chi.Ele.Pow.Pre,vol.5 ,pp.32-37,. June 2010.

[4] Z.M.Lin,Z.L.Cai, "Design and Realization of the ConfigurationTool Based on SCL Model." Pow.Sys.Pro.Con, vol.6,pp. 91-94, June 2009.

[5] W. H. Huang, Y. Lee, "Technology and Application of the IEC 61850 Standard”. Mod.Elec.Tech, vol.13, pp. 93-95, October 2011.

[6] W. J. Han, M. Kamber, "Data Ming Concepts and Techniques, Second Edition”. Chi. Mach.Pre, vol.21, pp. 23-26, October 007.

[7] L. X. Cao, H. Gao, "Based on Star Schema of a Multi-Channel Top - k Join Algorithm". Journal of computers, vol.35, pp. 34-10, May 2011 .

[8] Z.G.Lee,"Object-Oriented Database System Preliminary Discussion”. Chi. Man.Infor, vol.16, pp. 21-25, July 2013.

[9] C.S.Horstmann, "Gary Cornell. Core Java 2 Volume I Fundamentals". China Machine PRESS, vol.46, pp. 54-58, July 2002.

[10] C. F. Goldfarb, "Prescod P. XML Manual". Elect.Indus.Press, vol.46,pp. 2-15, June 2003,

[11] D. Lee, "Comparative Analysis of Six XML Schema Languages.' ACM SIGMOD Record, vol.32,pp. 117-151,May 2002.

[12] S. Sen, N. Chaki, "A Framework To Convert XML Schema to ROLAP". 2011 Second International Conference on Emerging Applications of Information Technology ,vol.22,pp. 26-30,May 2012. 